Ann. Sci. For., 1987, $44 \cdot(1), 85-102$

\title{
Etude de la sectorisation des souches de châtaignier (Castanea sativa Mill.) à l'aide d'eau tritiée
}

\author{
G. CARLIER \\ avec la collaboration technique de Christiane Dubois-Paganon \\ Université scientifique et médicale de Grenoble, \\ Laboratoire de Botanique, B.P. 68, \\ F 38402 Saint-Martin-d'Hères Cedex
}

\begin{abstract}
Résumé
La sectorisation de quatre souches de châtaignier, portant 5 à 7 rejets de cinq ans, a été étudiée par injection d'eau tritiée dans une racine et mesure de la radioactivité spécifique de l'eau transpirée par les différents rejets. L'expérience a été effectuée trois fois avec des racines différentes. Quelle que soit la racine qui a reçu l'injection, tous les rejets reçoivent de la radioactivité, mais les rejets les plus proches sont très significativement favorisés. On conclut que la sectorisation est seulement relative et que les secteurs de la souche alimentés préférentiellement par les différentes racines peuvent se chevaucher. Par contre il n'a pas été mis en évidence de préférence significative en faveur des rejets les plus vigoureux.
\end{abstract}

\section{Introduction}

Les forêts de châtaigniers sont le plus souvent traitées en taillis grâce à la propriété de cette essence de donner des rejets après coupe rase du pied-mère. Le nombre de rejets vivants décroît au cours de la rotation (Berthier, 1984). Certains exploitants pratiquent des éclaircies afin d'avantager les rejets les plus forts. L'avantage peut consister en une meilleure exposition à la lumière et en une alimentation accrue en sève brute. Ce deuxième avantage ne peut jouer que si la sève transitant par chaque racine est susceptible d'alimenter plusieurs rejets ou même tous. C'est là qu'on peut considérer l'éventualité d'une «sectorisation » des souches.

On dit qu'une souche est découpée en secteurs si la sève brute issue d'une racine alimente de préférence un ou plusieurs rejets à l'exclusion des autres. L'existence de secteurs indépendants a été décrite pour les souches de chêne-liège (DESTREMEAU \& Roderbourg, 1968). D'après Riedacker (1973), dans la souche jeune d'eucalyptus, la sève d'une racine passe à 90 p. 100 dans les rejets implantés dans le quadrant centré sur cette racine ; dans les souches âgées, chaque racine alimente encore préférentiellement un secteur, mais la position de celui-ci n'est plus fixée aussi rigoureusement. Chez le bouleau, Bedeneau \& PAges (1984) montrent l'existence de relations privilégiées entre certaines racines et certains "boutons" (ensembles de jeunes rejets groupés) 
mais ces relations ne peuvent pas être prédites d'après la proximité. Chez le châtaignier, d'après Aymard \& FREDOn (1986), " une racine alimente en priorité les rejets qui lui sont proches; mais lors de l'application de fortes doses de marqueur, la distribution s'étend à la sous-unité ou même à l'ensemble de la cépée ".

Les marqueurs utilisés par les auteurs cités sont des substances transportées par la sève brute : phytocides dont on observe les effets nocifs sur les rejets, ou phosphates radioactifs que l'on dose dans des échantillons après un temps choisi. La détection directe des phosphates radioactifs par des capteurs externes déplacés à la surface de l'écorce a été pratiquée sur des arbres de futaie (OWSTON et al., 1970, 1972) mais n'a pas été utilisée pour les souches de taillis. Les doses nécessaires seraient très élevées.

Dans le travail présenté ici, on utilise l'eau tritiée ${ }^{3} \mathrm{H}_{2} \mathrm{O}$, qui est évidemment le meilleur marqueur du mouvement de l'eau, princjpal constituant de la sève. L'objectif du travail est de rechercher, en fournissant de l'eau tritiée successivement à plusieurs racines d'une souche, s'il existe des relations privilégiées entre racines et rejets et quels sont les rejets avantagés, compte tenu de leur position et de leur vigueur.

\section{Techniques expérimentales et matériel utilisé}

\subsection{Matériel}

L'étude porte sur 4 souches d'un taillis de 5 ans (en 1984) à Notre-Dame de l'Osier (Isère). On désigne ici par "souche " un ensemble bien distinct, de 60 à $90 \mathrm{~cm}$ de diamètre au niveau du sol, comportant 5 à 8 "chicots" (bases de rejets de la rotation précédente) et 5 à 7 rejets vivants. Trois de ces souches sont encore reliées à des souches voisines, distantes de plusieurs décimètres ou mètres, par des isthmes ligneux en décomposition. L'origine du taillis est ancienne; le recépage y est pratiqué tous les 20-25 ans.

La base des souches a été dégagée de la souche superficielle de sol afin d'atteindre les racines périphériques et, dans la mesure du possible, les racines sous-jacentes. Les souches ont alors été dessinées (fig. 2). Les chicots appartenant à la rotation précédente sont aisément identifiables ; ceux des rotations antérieures le sont plus difficilement. La hauteur totale des rejets et leur circonférence à $1,30 \mathrm{~m}$ ont été mesurées le 26 juin et le 27 septembre 1984 (tabl. 1).

\subsection{Injections d'eau tritiée}

A chaque expérience, l'eau tritiée est injectée dans une seule racine, d'au moins $25 \mathrm{~mm}$ de diamètre. Trois injections dans des racines différentes ont été pratiquées sur chaque souche, respectivement fin juin, fin août et fin septembre 1984. Le choix s'est porté sur des racines aussi bien réparties que possible autour de chaque souche, à l'exception de celles qui sont nettement à l'écart de l'ensemble des rejets (fig. 2).

Un trou (diamètre : $3,5 \mathrm{~mm}$; profondeur : $7 \mathrm{~mm}$ ) est percé à la chignolle. Le fût d'une seringue de $1 \mathrm{ml}$ y est enfoncé à force et aussitôt rempli d'eau distillée afin de vérifier que celle-ci pénètre spontanément; simon la pénétration est amorcée en appliquant de brèves surpressions. On dépose alors dans la seringue, en plusieurs fois si 


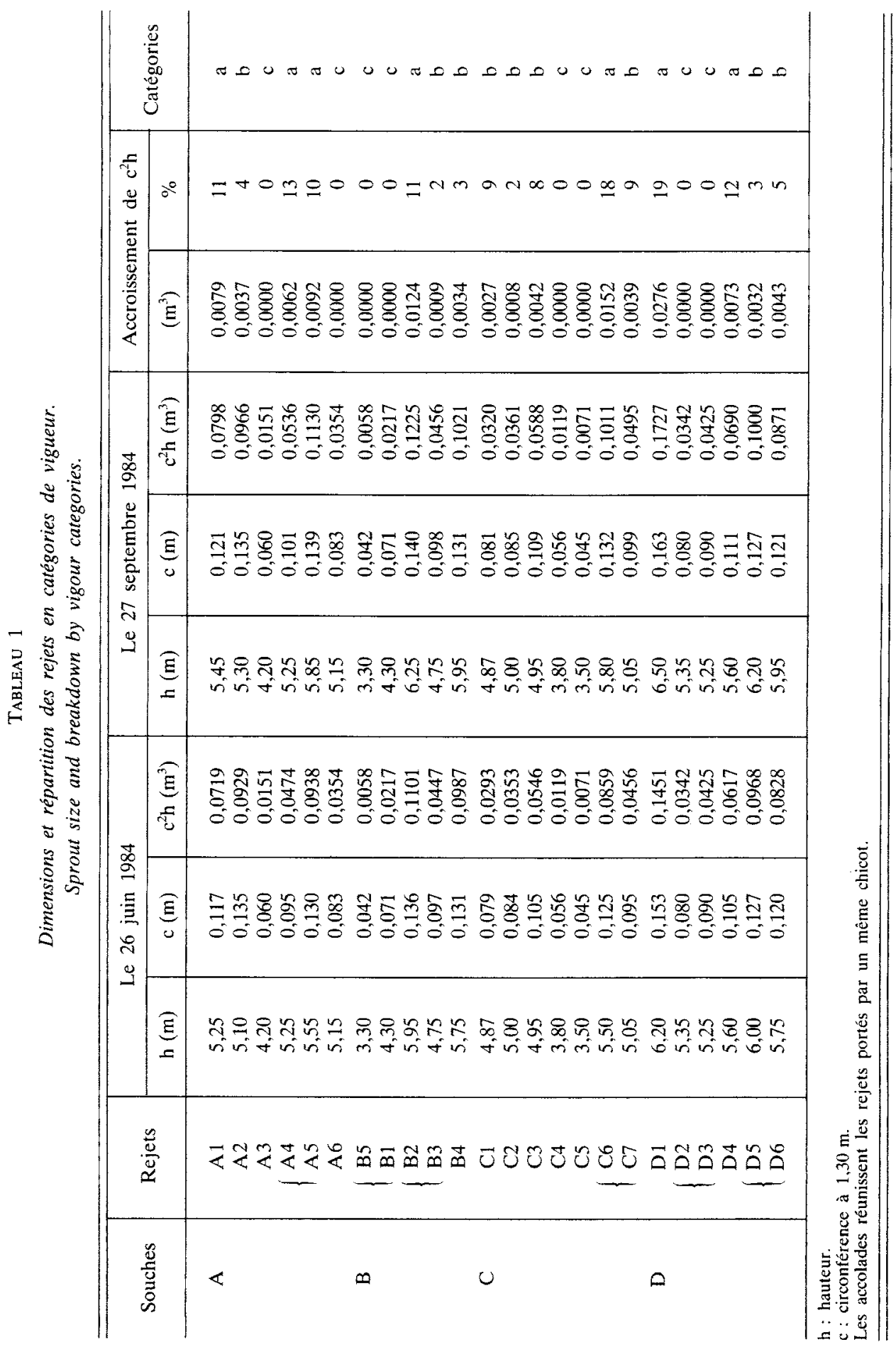


c'est nécessaire, le volume prévu d'eau tritiée (en général 1 ou $2 \mathrm{ml}$ ), de radioactivité spécifique $10^{9} \mathrm{dpm} \mathrm{ml} \mathrm{ml}^{-1}$. Après pénétration on effectue encore trois rinçages par $1 \mathrm{ml}$ d'eau distillée. La seringue est laissée en place et bouchée par son piston. Pour les grosses racines, l'eau tritiée est répartie entre deux ou trois trous.

\subsection{Echantillons d'eau transpirée}

La collecte d'eau transpirée est inspirée du Luvall \& MurPhy (1982). Dès l'injection un rameau feuillé de chaque rejet est coiffé d'un sac de polyéthylène ligaturé à la base, dans lequel l'eau transpirée se condense. Les sacs sont changés à intervalles de 2-4 jours et placés à chaque fois sur un rameau différent (cependant lorque le feuillage est mouillé de pluie, le même rameau sert deux fois de suite afin d'éviter de diluer l'eau radioactive transpirée). Les perturbations que cette procédure peut apporter à la transpiration sont discutées plus loin.

Suivant l'exposition du rameau, le volume recueilli à chaque récolte va d'une fraction de $\mathrm{ml}$ à une centaine de $\mathrm{ml}$. La collecte est poursuivie tant que la radioactivité spécifique de l'eau transpirée par les rejets les plus favorisés est supérieure à quelques milliers de $\mathrm{dpm} \mathrm{ml}^{-1}$; à ce moment celle des autres rejets est devenue négligeable. La durée totale de collecte peut atteindre 3 semaines si la transpiration est peu intense.

\subsection{Mesure de la radioactivité spécifique (dpm $\mathrm{ml}^{-1}$ )}

La radioactivité de parties aliquotes (par exemple $0,5 \mathrm{ml}$ ) est mesurée à l'aide d'un spectromètre à scintillation Tricarb (marque Packard), dans un mélange d'éthanol absolu $(4,7 \mathrm{ml})$ et de solution scintillante $(10 \mathrm{ml})$. La solution scintillante contient $4 \mathrm{~g}$ de 2,5-diphényloxazole (PPO) et $0,1 \mathrm{~g}$ de 1,4-bis- |2-(4-méthyl-5-phényloxazolyl) |benzène (diméthyl-POPOP) par litre de toluène.

\subsection{Calcul de la répartition de l'eau tritiée entre les rejets}

Soit $n$ le nombre de rejets de la souche et $p$ le nombre de périodes de collecte d'eau transpirée. Pendant la période $j$, de durée $t_{\mathrm{j}}$, le rejet $i$ a une intensité transpiratoire moyenne $T r_{i j}$ et l'eau transpirée a une radioactivité spécifique $r s_{i j}$. La quantité de radioactivité $r_{i j}$ (en dpm) qui a atteint ce rejet durant la période $j$ est :

$$
\mathbf{r}_{\mathrm{ij}}=\mathrm{rs}_{\mathrm{ij}} \times \mathrm{t}_{\mathrm{j}} \times \mathbf{T r}_{\mathrm{ij}}
$$

On ne connaît pas la valeur de $T r_{i j}$. Il faut donc l'estimer indirectement. La transpiration d'un rejet doit dépendre à la fois de ses caractéristiques biométriques et des conditions météorologiques. En admettant que la transpiration est proportionnelle à l'aire de la section conductrice (mesurée à $1,30 \mathrm{~m}$ ) $S_{i}$ et à l'évapotranspiration potentielle journalière moyenne durant la période $j, E T P_{j}$, on peut remplacer $\operatorname{Tr}_{i j}$ par $k S_{i} E T P_{j}$.

L'ETP est calculée par la méthode de Brochet \& Gerbier (1972) qui donne des résultats moyens par rapport à d'autres méthodes classiques. La détermination de $S_{i}$ est décrite au paragraphe 6 ci-dessous.

Il vient alors :

$$
r_{i j}=r s_{i j} t_{j} k S_{i} E_{j}
$$


La quantité totale de radioactivité $R i$ reçue par le rejet $i$ est la somme des radioactivités reçues durant les périodes $l$ à $p$ :

$$
R_{i}=k S_{i} \sum_{j=1}^{p}\left(r_{i j} t_{j} E^{2} P_{j}\right)
$$

La somme des quantités de radioactivité reçues par les rejets $l$ à $n$ est la radioactivité totale transportée à partir de la racine injectée, $R_{t o t}$ :

$$
R_{t o t}=k \sum_{i=1}^{n}\left(S i \sum_{j=1}^{p}\left(r s_{i j} t_{j} E_{T P}\right)\right)
$$

et la part $P$ reçue par le rejet $i$, en pourcentage du total, est :

$$
P=100 R_{i} / R_{\text {tot }}
$$

expression qui élimine $k$. On admet que $k$ est bien une constante, ce qui sera discuté plus loin.

On considère que la répartition de la radioactivité représente celle du flux d'eau, et donc de la sève brute, passant par la racine injectée.

\subsection{Estimation de l'aire de la section conductrice $S_{i}$}

Comme les rejets doivent être préservés en vue d'expériences ultérieures, cette aire doit être calculée à l'aide d'un tarif qui la relie à la circonférence.

Vingt rejets de 5 ans, de circonférence comprise entre 44 et $169 \mathrm{~mm}$ ont été récoltés dans le même taillis que les souches expérimentales. Des tranches prélevées à $1,30 \mathrm{~m}$ ont été traitées au jaune de diméthyle qui colore le bois de cour en rouge, ou au Lugol qui permet de localiser le bois fonctionnel, dont le parenchyme accumule de l'amidon. Les deux colorations indiquent que le bois d'aubier est constitué par les cernes des trois dernières années.

L'aire de l'ensemble de ces 3 cernes a été calculée à partir d'au moins 9 mesures des diamètres interne et externe, et le tarif fournissant la meilleure corrélation entre l'aire $S$ et une ou plusieurs fonctions de la circonférence $c$ a été recherché. Le tarif retenu est :

$$
S=a+b_{1} c^{1,5}+b_{2}(\log c)^{2,3}
$$

où $S$ est en $\mathrm{mm}^{2}$ et $c$ en $\mathrm{mm}$, avec :

$$
\begin{aligned}
& a=177,041 \\
& b_{1}=0,967 \\
& b_{2}=-120,000
\end{aligned}
$$

Le coefficient de corrélation double est $r=0,9950$. Grâce à ce tarif, l'aire $S_{\mathrm{i}}$ est calculée pour chaque rejet le 26 juin (valeur applicable à la première expérience) et le 27 septembre (valeur applicable aux deux autres expériences).

\subsection{Classement hiérarchique des rejets (tabl. 1)}

Dans l'hypothèse où la répartition de la sève serait influencée par la vigueur des rejets, il était nécessaire de caractériser celle-ci. L'observation permet de distinguer les rejets « dominés » (entièrement sous le couvert des autres) et les "dominants » (les plus forts de chaque souche), les autres pouvant être qualifiés de «moyens». Mais 
cette distinction n'est pas sans subjectivité. Le classement adopté ici repose sur le fait que les rejets les plus vigoureux ont la croissance en hauteur et circonférence la plus forte et la plus prolongée dans la saison : d'après l'accroissement du produit $c^{2} h$ entre le 26 juin et le 27 septembre, les rejets sont classés en trois catégories :

- catégorie $a$ : accroissement de 10 p. 100 et plus,

- catégorie $b$ : accroissement de 1 à 9 p. 100 ,

- catégorie $c$ : accroissement nul.

L'effectif total des rejets (24) se répartit en 7 de catégorie $a, 9$ de catégorie $b$ et 8 de catégorie $c$. Le classement n'est pas modifié si on prend pour critère l'accroissement absolu plutôt que l'accroissement relatif (tabl. 1).

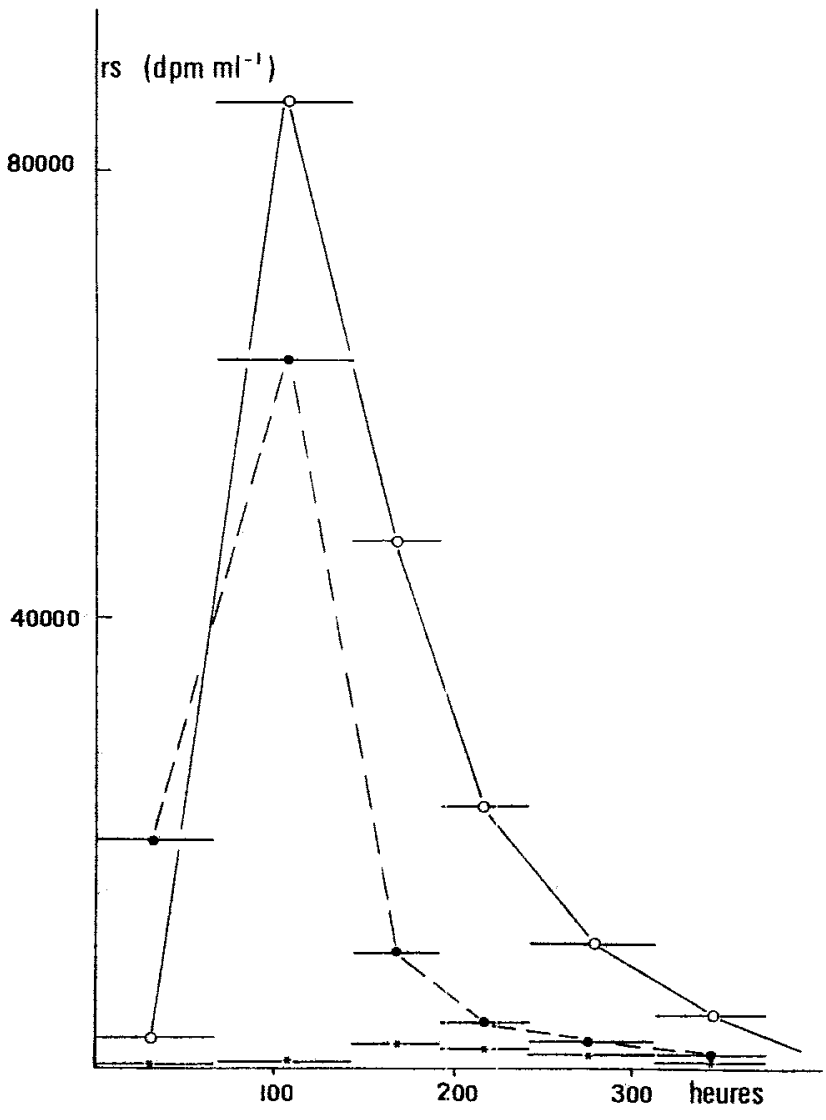

FiG. 1

Evolution de la radioactivité spécifique de l'eau transpirée par les trois rejets les plus fortement marqués de la souche B à la suite de l'injection d'eau tritiée du 26 juin 1984.

Time-course of the specific radioactivity of water transpired from the three sprouts most strongly labelled of stump B, following injection of radioactive water on june 26th 1984. 


\section{Résultats}

\subsection{Evolution de la radioactivité spécifique de l'eau transpirée}

A titre d'exemple, la figure 1 montre l'évolution de la radioactivité spécifique de l'eau collectée à partir des trois rejets les plus fortement marqués de la souche $\mathrm{B}$, à la suite de l'injection de juin. La radioactivité spécifique de l'eau transpirée par les autres rejets, sans être nulle, est trop faible pour être représentée sur la figure. Les barres horizontales indiquent les durées des collectes $t_{i j}$ et l'ordonnée des points représente $r s_{i j}$ (termes intervenant dans les équations 1 à 4). Dans le cas représenté, la collecte a été arrêtée 360 heures (15 jours) après l'injection. La radioactivité spécifique de la dernière collecte était tombée à $4350 \mathrm{dpm} \mathrm{m}{ }^{-1}$ pour le rejet le plus fortement marqué ; la poursuite des collectes n'aurait évidemment pas modifié le résultat des calculs. Par contre, il faut éviter l'interférence d'une expérience sur la suivante, car un rejet très marqué lors de la première peut l'être très peu à la seconde. Il est donc nécessaire de laisser l'élimination de l'eau tritiée dans l'atmosphère se poursuivre durant un intervalle de sécurité d'au moins encore 100 heures. Les durées de collecte et de sécurité doivent être allongées si l'ETP est réduite.

\subsection{Répartition du flux de sève à partir des racines injectées (tabl. 2 et fig. 2)}

Les racines injectées à chaque expérience sont désignées sur la figure 2. Le tableau 2 montre que la radioactivité est décelée dans l'eau transpirée par tous les rejets, quelle que soit la racine injectée. Mais la répartition est toujours très inégale, puisque sur les 12 expériences ( 4 souches $\times 3$ injections) :

- dans 12 cas, au moins 50 p. 100 de la radioactivité est reçue par 1 rejet,

- dans 8 cas, au moins 75 p. 100 de la radioactivité est reçue par 1 rejet,

- dans 5 cas, au moins 90 p. 100 de la radioactivité est reçue par 1 rejet.

Dans tous les cas, trois rejets au maximum se partagent 90 p. 100 du flux. En conséquence sur les 72 individus ( 24 rejets $\times 3$ injections) 32 reçoivent chacun moins de 1 p. 100 du flux. Dans la suite on appellera « rejet le plus favorisé » celui qui reçoit la plus grande part du flux et « rejets favorisés » ceux qui reçoivent au moins 10 p. 100 du flux issu d'une racine.

La figure 2 fait apparaître les relations privilégiées. Trois rejets ( 2 rejets $a$ et un rejet $b$ ) ont été favorisés à partir de différentes racines mais un seul a été le plus favorisé à partir de deux racines.

\subsection{Effet de la proximité}

La figure 3 fait apparaître la relation entre la part de flux reçue par un rejet et sa distance par rapport à la racine injectée. Cette distance est mesurée horizontalement entre l'aplomb du raccord de la racine sur la souche et l'axe du rejet. Au-delà de $30 \mathrm{~cm}$ la part est toujours inférieure à 10 p. 100 . Mais en deçà de cette distance, la dispersion est considérable.

De toute façon la valeur absolue de la distance ne peut pas être prise comme critère d'un traitement statistique, car les souches sont de dimensions différentes et la distance d'une racine au rejet le plus proche (par exemple) varie considérablement, de 

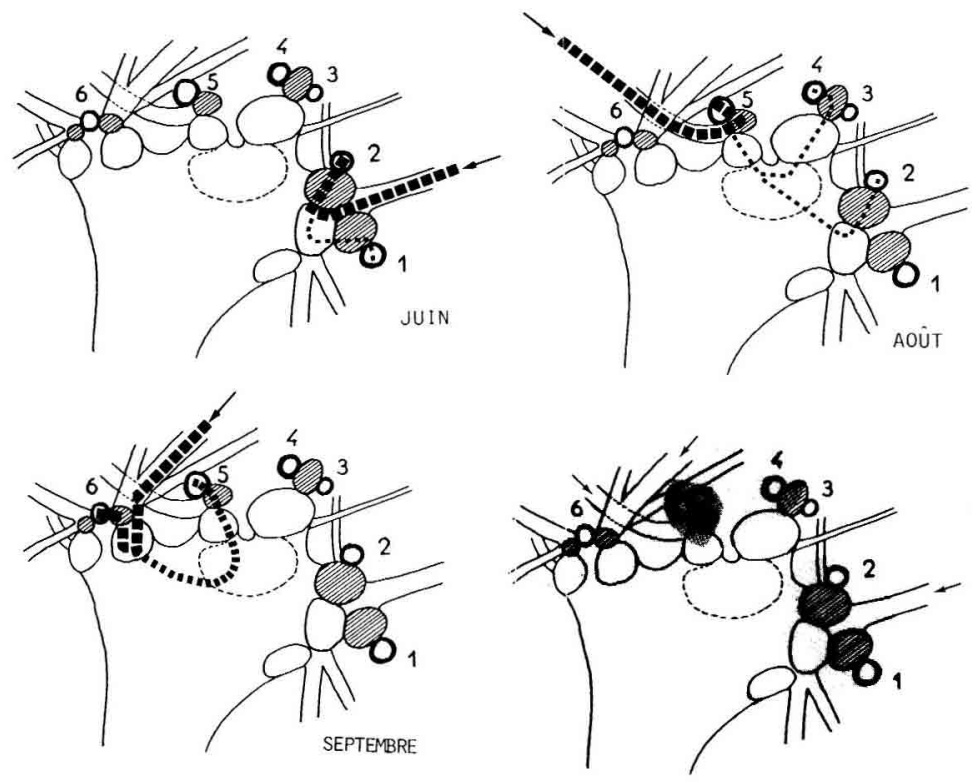

A
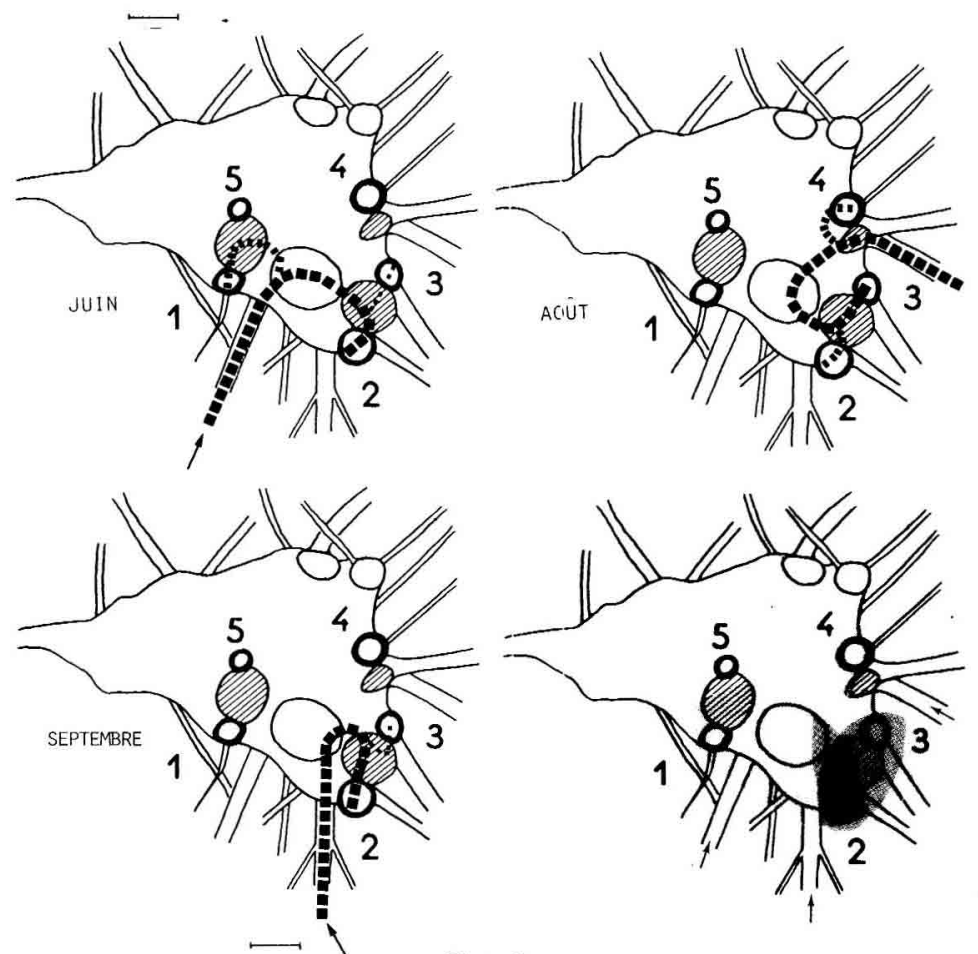

FIG. 2

Répartition de l'eau tritiée et sectorisation des souches $A, B, C, D$. Contours gras : rejets actuels, numérotés (rotation $\mathrm{n}$ ); contours maigres et hachures : chicots de la rotation $\mathrm{n}-1$;

contours maigres : chicots de la rotation $\mathrm{n}-2$; contours en tirets : chicots de la rotation $\mathrm{n}-3$. Les tracés indiquent les pourcentages du flux de sève: tracé large, plus de $50 \mathrm{p} .100$; tracé moyen, 10 à $49 \mathrm{p}$. 100 ; tracé étroit, l à 9 p. 100 . Les zones ombrées recouvrent les secteurs proposés pour chaque racine injectée.

Distribution of radioactive water and sectorization of stumps $A, B, C$ and $D$. Thick outlines : existing, numbered sprouts (rotation $\mathrm{n}$ ); thin outlines and hatching: stumps from rotation $\mathrm{n}-1$,

thin outlines: stumps from rotation $\mathrm{n}-2$; dashed outlines: stumps from rotation $\mathrm{n}-3$.

Sketches indicate the sap flow percentages : broad sketch, more than 50 p. 100 ; mediumsized sketch : $10-49$ p. 100 ; narrow sketch : 1-9 p. 100. Shadowed zones cover sectors proposed for each injected root. 

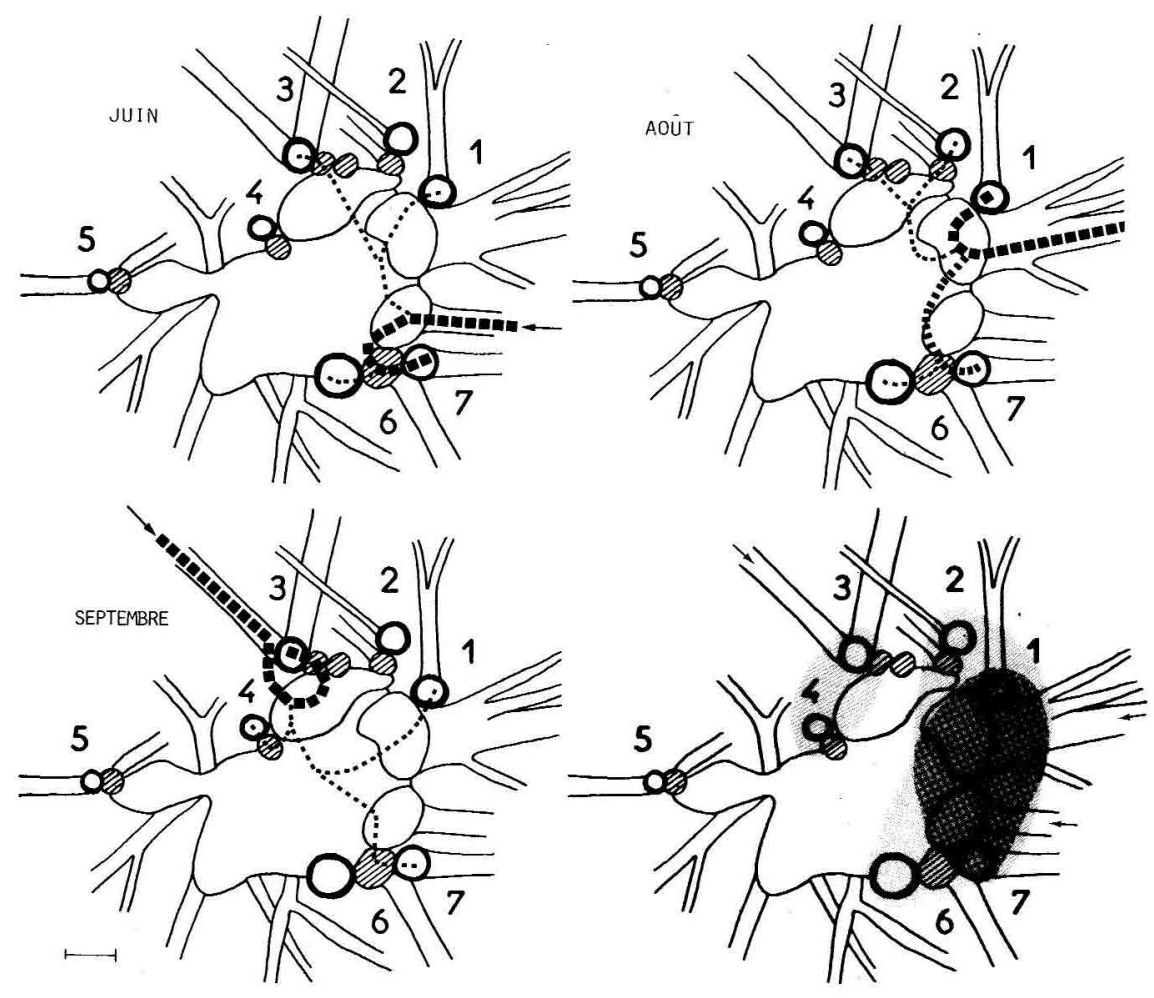

C

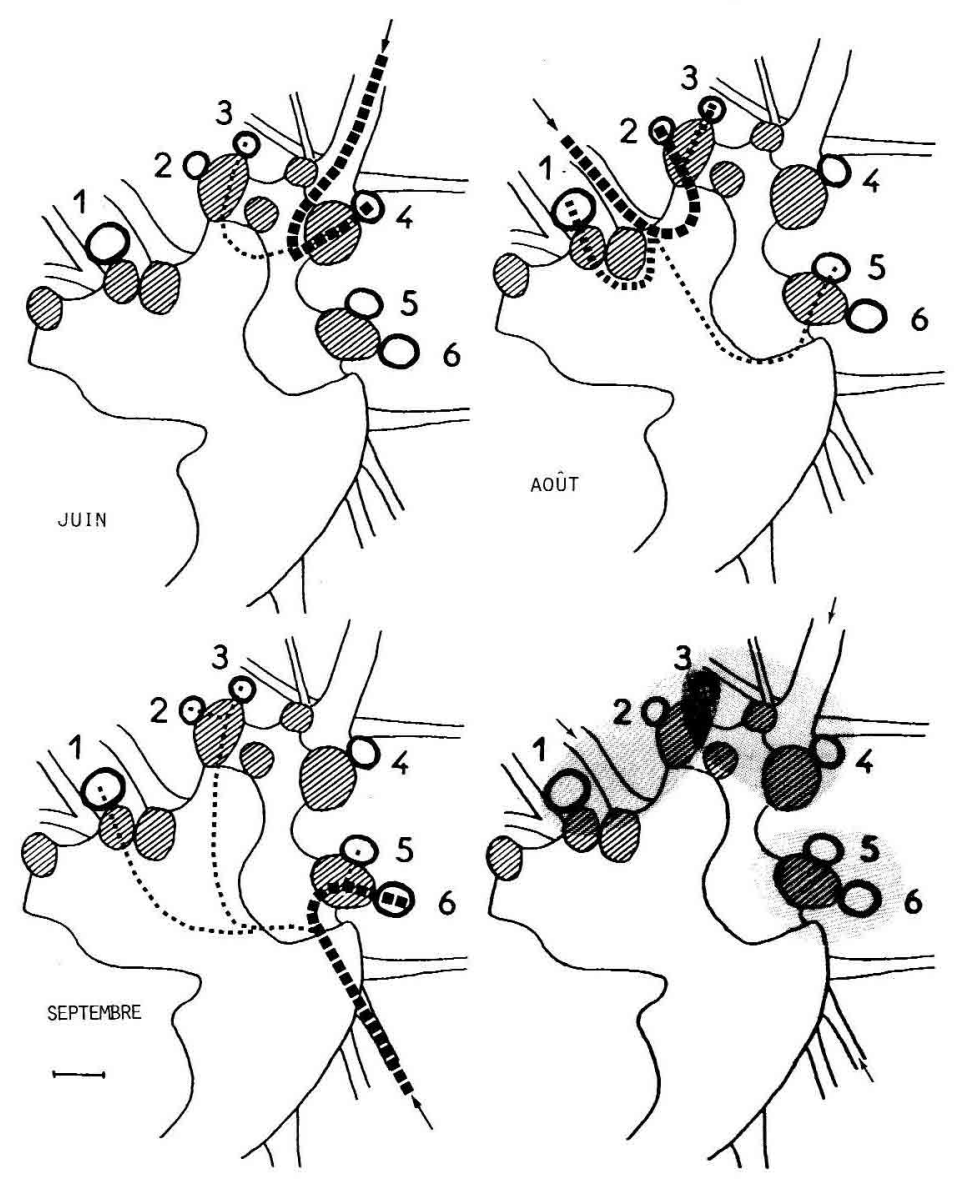




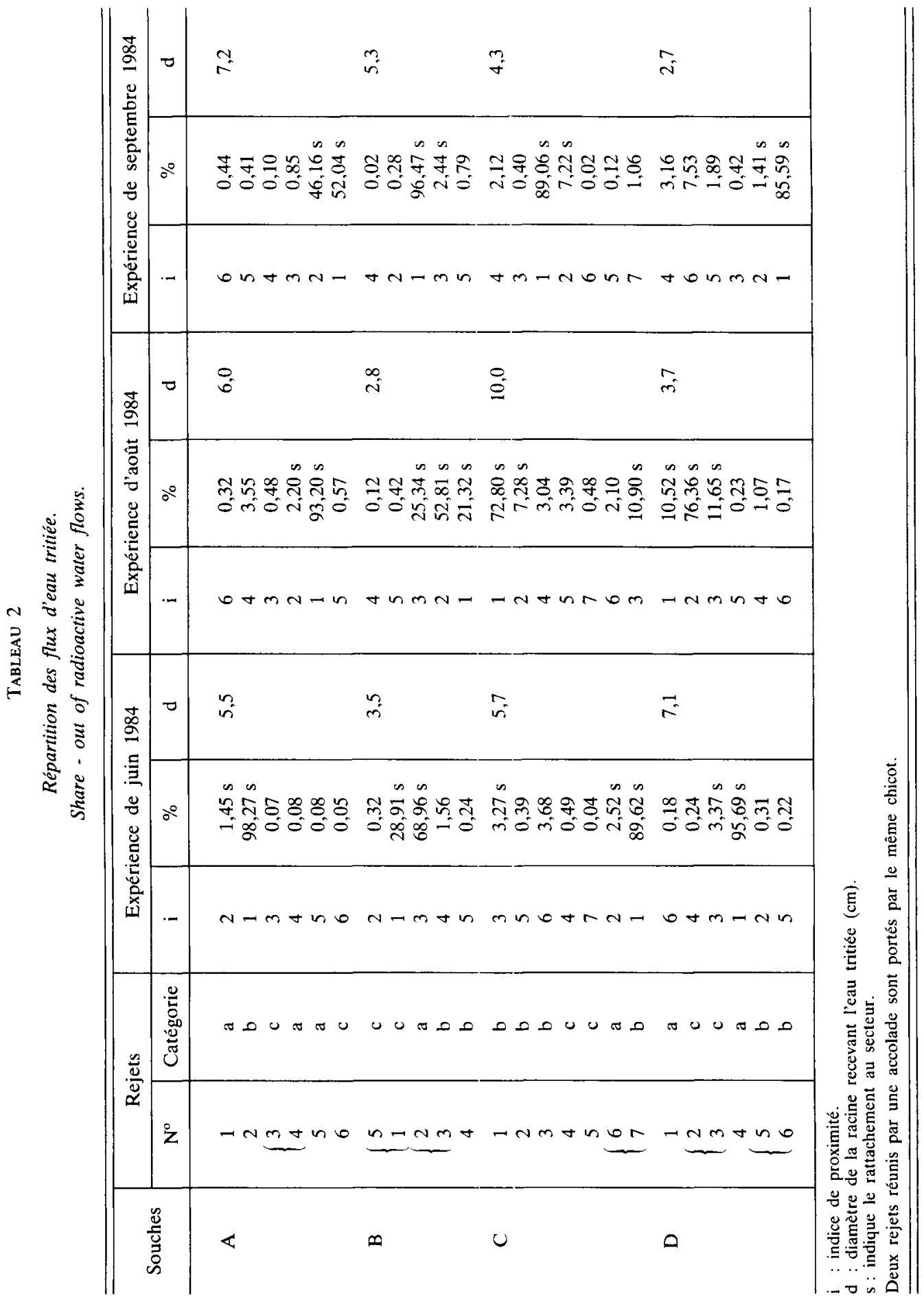




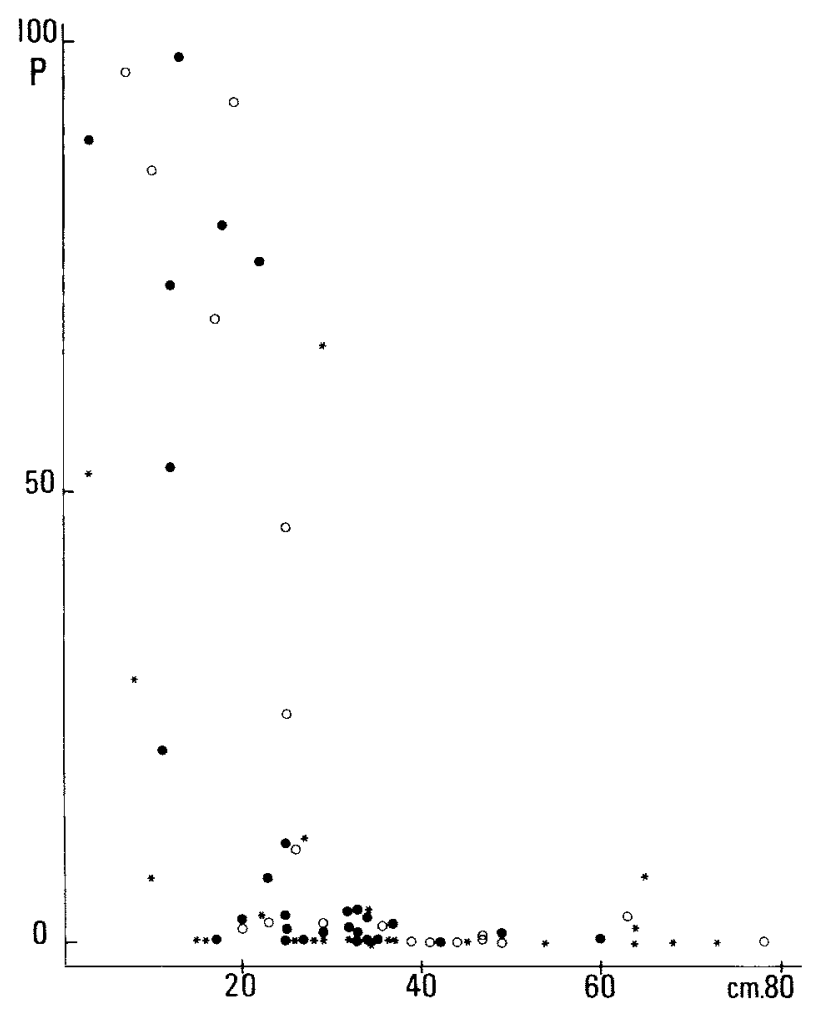

FIG. 3

Relation entre le pourcentage de flux de sève $\mathrm{P}$ reçu par les rejets et la distance de ceux-ci à la racine ayant reçu l'injection d'eau tritiée.

Relation between the percentage of sap flow received by a sprout and the distance from the sprouts to the root fed with radioactive water.

- Rejets de catégorie $a$. - a sprouts.

Rejets de catégorie $b$. - $b$ sprouts.

* Rejets de catégorie $c$. - c sprouts.

3 à $26 \mathrm{~cm}$. Ce qui importe est plutôt le rang de chaque rejet dans l'ensemble des distances. Pour chaque expérience, les rejets sont donc affectés d'un indice de proximité égal à ce rang, l'indice 1 étant attribué au rejet le plus proche.

Le tableau 2 montre alors que le rejet le plus favorisé est :

- dans 9 cas le rejet de rang 1 ,

- dans 2 cas le rejet de rang 2 ,

- dans 1 cas le rejet de rang 3 .

La démonstration statistique de l'effet de la proximité peut être faite en répartissant les 72 individus ( 24 rejets $\times 3$ expériences) en classes de proximité : les rejets de rang 1 forment la classe I (effectif : 12), ceux de rang 2 la classe II (effectif : 12) et ceux de rangs 3 et au delà de la classe III (effectif : 48). Des essais de répartition en un plus grand nombre de classes se sont avérés, après traitement statistique, dépourvus d'intérêt. 
Tableau 3

Pourcentage moyen du flux de sève reçu par les rejets suivant leur classe de proximité et leur catégorie de vigueur.

Mean percentage of sap flow received by the sprouts according to proximity classes and vigour cutegories.

\begin{tabular}{|c|c|c|c|c|c|}
\hline \multirow{2}{*}{\multicolumn{2}{|c|}{$\begin{array}{c}\text { Classes } \\
\text { de proximité }\end{array}$}} & \multicolumn{4}{|c|}{ Cattégories } \\
\hline & & a & b & c & Ensemble \\
\hline I & $\begin{array}{r}\mathbf{n} \\
\mathrm{m}(\mathrm{s})\end{array}$ & $\begin{array}{c}4 \\
74,0(42,3)\end{array}$ & $\begin{array}{c}6 \\
76,1 \\
(28,1)\end{array}$ & $\begin{array}{c}2 \\
40,5(16,4)\end{array}$ & $\begin{array}{c}12 \\
69,5(32,5)\end{array}$ \\
\hline II & $\begin{array}{r}n \\
m(s)\end{array}$ & $\begin{array}{c}4 \\
13,1\end{array}$ & $\begin{array}{c}4 \\
15,5\end{array}$ & $\begin{array}{c}4 \\
21,0 \\
(37,0)\end{array}$ & $\begin{array}{c}12 \\
16,6(26,4)\end{array}$ \\
\hline III & $\begin{array}{r}\mathbf{n} \\
\mathrm{m}(\mathrm{s})\end{array}$ & $\begin{array}{c}13 \\
7,9(19,6)\end{array}$ & $\stackrel{17}{2,1(2,6)}$ & 18 & $\begin{array}{c}48 \\
3,5(10,5)\end{array}$ \\
\hline Ensemble & $m(s)^{n}$ & $\begin{array}{c}21 \\
21,5(35,5)\end{array}$ & $\begin{array}{c}27 \\
20,5(34,2)\end{array}$ & $\begin{array}{c}24 \\
8,2(18,7)\end{array}$ & 72 \\
\hline \multicolumn{6}{|c|}{$\begin{array}{l}\text { n : effectif. } \\
\text { m : moyenne. } \\
\text { s : écart-type. }\end{array}$} \\
\hline
\end{tabular}

Le tableau 3 fait apparaître les pourcentages de flux moyens reçus par les individus des trois classes de proximité, le classement étant fait soit dans l'ensemble de tous les rejets, soit séparément pour les trois catégories de rejets. Dans tous les cas, le pourcentage de flux moyen décroît quand on considère les classes de proximité décroissante (I à III) mais comme dans chaque classe la dispersion est considérable il faut rechercher si cet effet est significatif. Or l'analyse de variance est illicite, car au moins l'une des conditions de validité n'est pas remplie : les variances ne sont pas homogènes, sauf exception. On a donc utilisé un test non paramétrique, celui de Kruskall-Wallis (SoKal \& RoHLF, 1981).

Les résultats du test figurent au tableau 4. L'effet de la proximité est hautement significatif si l'on effectue le test sur l'ensemble des 72 individus, ou sur l'ensemble des individus de catégorie $a$ (les plus vigoureux) ou de catégorie $b$. Par contre l'effet n'est pas significatif si on ne considère que l'ensemble des individus de catégorie $c$ (les moins vigoureux); mais cela paraît dû à ce que, dáns cette catégorie, seulement deux individus se sont trouvés dans la classe de proxirnité $I$.

Il faut souligner que l'effet de proximité n'est pas absolu. Il arrive que le rejet le plus proche de la racine injectée ne soit pas le plus favorisé, mais il peut toujours être qualifié de «favorisé », c'est-à-dire qu'il reçoit au moins 10 p. 100 du flux (tableau 2 : souche $B$, expériences de juin et d'août ; souche $D$, expérience d'août).

L'effet de proximité se fait encore sentir si l'on compare des rejets « frères » c'està-dire portés par un même chicot (tableau 2). Dans 14 cas sur 18, c'est le « frère »le plus proche de la racine injectée qui reçoit la part la plus importante des deux; l'inverse n'est réalisé que dans 4 cas sur 18. Cette distribution diffère significativement de celle qui correspondrait théoriquement à l'absence d'effet (c'est-à-dire 9 et 9), comme le montre par exemple un test G (Sokal \& Rohlf, 1981). Ceci indique qu'en transitant par un chicot, la sève ne se répartit pas uniformément autour de celui-ci, ce qui aurait pour effet d'alimenter également les rejets « frères». 
TABleaU 4

Résultats du test de Kruskall-Wallis.

Results of Kruskall-Wallis test.

\begin{tabular}{l|l|c|c|c}
\hline \hline \multicolumn{1}{c|}{ Facteur étudié } & $\begin{array}{c}\text { Rejets concernés } \\
\text { par le test }\end{array}$ & Effectif & Résultat & Observations \\
\hline Proximité & Toutes catégories & 72 & $* *$ & Entre I et II : ** \\
& Catégorie a & 21 & $* *$ & Entre II et III : * \\
& Catégorie b & 27 & NS & \\
Catégorie c & 24 & NS & NS & \\
Catégorie & Toutes classes & 72 & NS & \\
& Classe I & 12 & 12 & \\
& Classe II & 48 & Classe III & \\
& ** effet hautement significatif. & \\
* effet significatif. \\
NS non significatif. \\
\hline \hline
\end{tabular}

\subsection{Division des souches en secteurs}

Le tableau 2 montre qu'au rejet le plus favorisé on peut toujours adjoindre un rejet voisin, ou bien deux rejets voisins (qui sont alors situés de part et d'autre du rejet le plus favorisé). Cet ensemble reçoit au moins 87 p. 100 du flux (et en fait plus de 90 p. 100 dans 11 cas sur 12) : on peut le considérer comme le "secteur" de la racine injectée. Les rejets rattachés au secteur d'une racine sont signalés dans le tableau 2 et les secteurs sont localisés sur la figure 2. Pour chaque souche on observe le chevauchement d'au moins deux secteurs. Pour la souche $\mathrm{B}$, dont les rejets sont nettement plus groupés que ceux des autres souches, les trois secteurs identifiés se chevauchent.

\subsection{Recherche d'un effet de la vigueur des rejets}

Les rejets ayant été répartis en catégories $(a, b, c)$ d'après leur vigueur, on peut comparer les pourcentages de flux reçus par chaque catégorie, soit sans tenir compte de la proximité, soit séparément à l'intérieur de chaque classe de proximité. Le tableau 3 montre que les pourcentages de flux moyens vont en décroissant quand on passe des rejets les plus vigoureux (a) aux moins vigoureux (c), sauf dans la classe de proximité II. Mais en aucun cas cet effet n'est significatif (tabl. 4).

On remarque en outre que le rejet le plus favorisé appartient 4 fois sur 12 à la catégorie $a, 6$ fois sur 12 à la catégorie $b$ et 2 fois à la catégorie $c$. On peut rechercher si cette distribution diffère significativement de celle des rejets dans les trois catégories, c'est-à-dire 7,9 et 8 sur 24 soit $3,5,4,5$ et 4 sur 12 . Un test de conformité montre que les deux distributions ne diffèrent pas significativement. Donc on ne peut pas dire qu'une catégorie de rejets soit systématiquement plus favorisée, ou moins favorisée que les autres. 


\subsection{Recherche d'un effet de la taille des racines injectées}

Le diamètre des racines injectées va de 2,7 à $10 \mathrm{~cm}$ (au niveau du raccord à la souche). Deux hypothèses ont été éprouvées : a) les plus grosses racines alimenteraient préférentiellement les rejets les plus vigoureux, et b) les plus grosses racines alimenteraient le plus grand nombre de rejets, ce qui se traduirait par une fréquence élevée de rejets recevant un pourcentage de flux au moins égal à une valeur choisie comme seuil.

Pour éprouver l'hypothèse $a$ on a recherché s'il existe une corrélation significative entre le diamètre des racines et la valeur de $c^{2} h$ des rejets les plus favorisés, ou entre le diamètre des racines et l'accroissement de $c$ 'h des rejets les plus favorisés. Pour éprouver l'hypothèse $b$ on a cherché s'il y a une corrélation significative entre le diamètre de la racine et la fréquence des rejets recevant au moins 1 p. 100 ou 5 p. 100 ou 10 p. 100 du flux.

Dans aucun cas il n'a été trouvé de corrélation significative.

\section{Discussion}

\subsection{Validité de la méthode}

La transpiration $\operatorname{Tr}_{i j}$ est un terme indispensable au calcul de la radioactivité atteignant un rejet $i$. Or il était impossible de la déterminer expérimentalement. La méthode du transfert d'eau tritiée (KLINE et al., 1970) est évidemment applicable aux rejets ; mais il est exclu que l'on injecte de l'eau tritiée à la fois dans une racine, pour en rechercher la répartition entre les rejets, et dans les rejets, pour mesurer leur transpiration. La méthode du transfert de chaleur (Granier, 1985) est d'un développement trop récent pour qu'il ait été envisageable de l'appliquer en 1984.

Il fallait donc remplacer $T r_{i j}$ par une expression équivalente. Le fait que la transpiration soit proportionnelle à l'aire de la section conductrice a été démontré par KLine et al. (1976) pour le Douglas, et par JoRdan \& KLINE (1977) pour un ensemble d'espèces tropicales. Cette proportionalité sert souvent de base au calcul de la transpiration (Ibrahim et al., 1980; Granier, 1985 ; Bobay, 1985). Quant à la correspondance entre transpiration et ETP, elle a été montrée pour un peuplement de Pinus pinea à l'échelle du mois (Ibrahim et al., 1980) et pour des individus de Douglas, à l'échelle de la journée (Granier, 1985). Il faut noter qu'il n'est pas requis, pour la validité de la présente méthode, que le coefficient de proportionnalité entre transpiration et ETP soit le même pour toutes les souches ni pour toutes les expériences. Il suffit qu'il soit constant pour les différents rejets d'une souche et pour une expérience donnée. Il est possible que les rejets dont le feuillage est entièrement sous le couvert des aütres (A3, B5, C4 et C5) ne satisfassent pas à cette condition. Mais comme leur part de flux est faible, cette cause d'erreur ne peut invalider les conclusions positives concernant l'effet de la proximité. On peut cependant suspecter que si la part de flux reçue par ces rejets, qui appartiennent à la catégorie c (celle des moins vigoureux), a été surestimée, cela a pu empêcher de mettre en évidence de façon significative un éventuel effet de la vigueur des rejets. 
Un autre point essentiel à la validité de la méthode est que la présence des sacs plastiques ne doit pas modifier la radioactivité spécifique de l'eau collectée. Ce point a été discuté par Luvall \& Murphy (1982). Ceux-ci considèrent la collecte d'eau dans un sac comme un simple procédé d'extraction d'échantillons d'eau de l'arbre (ici : du rejet). Ainsi le flux effectivement transpiré par le rameau dans le sac n'a pas d'influence sur la radioactivité spécifique de leau, même si, comme il est probable, le sac modifie la transpiration du rameau. Cependant le rameau ne doit représenter qu'une petite fraction du feuillage du rejet, sous peine de modifier la transpiration totale de celui-ci. Cette condition peut ne pas avoir été satisfaite, en septembre 1984 , pour les rejets $\mathrm{C} 4$ et $\mathrm{C} 5$ qui avaient perdu une forte proportion de leur feuillage.

\subsection{Avantages et contraintes de la méthode}

Compte tenu des limitations énoncées ci-dessus, la méthode peut être créditée des avantages suivants : a) elle n'esț pas destructive, ni par prélèvements, ni par toxicité ; b) la totalité du flux de marqueur est prise en compte ; on ne risque pas de manquer le pic de radioactivité spécifique (fig. 1), comme le soulignent Luvall \& Murphy (1982); c) grâce à la longue période du tritium $(12,26$ ans) il n'y a pas de contraintes liées à la décroissance de la radioactivité ; d) le marqueur étant éliminé par la transpiration, plusieurs injections peuvent être faites au cours d'une même saison de façon à explorer les secteurs de plusieurs racines.

Par contre l'utilisation d'un radioisotope dans le milieu naturel est soumise à des conditions légales ${ }^{(1)}$. Compte tenu de la durée d'élimination, de la vitesse moyenne du vent sur le site et de la section du houppier, il faut calculer la quantité de radioactivité injectée pour que la dose maximale réalisée dans l'atmosphère soit inférieure à la dose admissible $\left(200 \mathrm{nCi} \mathrm{m}^{-3}\right)^{(2)}$.

\subsection{Relations préférentielles}

L'existence de telles relations a été mise en évidence dans tous les cas. Elles sont fondées significativement sur la proximité ; les trajets privilégiés sont généralement les plus courts, mais pas de façon absolue. Ces conclusions sont identiques à celles qui ont été obtenues par d'autres méthodes par Bedeneau \& Pages (1984) pour le bouleau et par Aymard \& Fredon (1986) pour le châtaignier.

Une répartition des rejets en secteurs a été tentée. S'il est assez aisé de désigner les rejets qui font partie du secteur d'une racine (le rejet le plus favorisé et son ou ses deux voisins immédiats), il est plus difficile et, en fait, arbitraire de désigner ceux qui n'en font pas partie, puisque tous les rejets reçoivent au moins une petite part du flux de radioactivité. Or même avec une délimitation restrictive des secteurs, on constate qu'il existe des chevauchements. Donc, s'ils existent, les secteurs ne sont pas indépendants ; chaque racine alimente plusieurs rejets et chaque rejet est alimenté par plusieurs racines. Il n'y a pas de strict découpage fonctionnel de la souche. C'est ainsi qu'AYMARD \& FREDON (1986) constatent que si l'on fournit une dose suffisante de phytocide, tous les rejets d'une souche sont atteints. Chez l'Eucalyptus au contraire, RiedAcker

(1) En France, l'autorisation esf donnée par la Commission Interministérielle des Radioéléments, B.P. 8, 91190 Gif-sur-Yvette.

(2) Décret 66-450 du 20 juin 1966, annexe IV, tableau 1. 
(1973) a conclu à l'indépendance des secteurs, non pas sur la base d'injections pratiquées dans différentes racines d'une même souche, mais du fait que l'ablation des rejets d'un secteur n'entraine pas la redistribution du marqueur vers d'autres secteurs. Chez le châtaignier, un dépressage devrait entraîner une redistribution du flux d'eau tritiée, ce qui devra être vérifié.

Il est surprenant que les rejets les plus vigoureux n'apparaissent pas significativement privilégiés. En raison de leurs dimensions et de l'extension de leur feuillage, ils sont le siège d'un fort flux de sève. On s'attendrait à ce qu'ils reçoivent les pourcentages de flux les plus élevés, ou à ce qu'ils soient les plus favorisés à partir du plus grand nombre de racines, ou encore à ce qu'ils soient alimentés par les plus grosses racines. Or aucun de ces effets n'apparaît de façon significative. Cela peut être dû au nombre insuffisant d'expériences ; ou bien le calcul des pourcentages de flux a pu être faussé à l'avantage des rejets les moins vigoureux, comme cela a été discuté plus haut. Toutefois, il faut noter que la méthode utilisée permet de connaître la répartition, en pourcentage, du flux passant par une racine, mais non la valeur absolue de ce flux. Il n'est donc pas exclu que les racines qui alimentent préférentiellement les rejets les plus vigoureux soient simplement le siège d'un flux de sève particulièrement élevé.

\title{
5. Conclusions
}

Les 4 souches de châtaignier étudiées ici. portant des rejets de 5 ans, ne peuvent pas être découpées en secteurs indépendants, mais il existe des relations préférentielles, généralement très marquées, entre racines et rejets. Ces relations sont fondées essentiellement sur la proximité. Le caractère relatif de la sectorisation suggère qu'en cas d'éclaircie les relations sont susceptibles d'être modifiées au bénéfice des rejets laissés sur pied.

Reçu le 19 juin 1985.

Accepté le 3 juin 1986.

\author{
Summary \\ A study of sectorization in Sweet-Chestnut (Castanea sativa Mill.) \\ stumps using radioactive water
}

Sectorization in four Sweet-Chestnut stumps, each bearing 5 to 7 five year old sprouts, was studied by the injection of radioactive water into a roct and the consequent measurement of the specific activity of the water transpired from each sprout. Each experiment was performed 3 times using different roots.

Regardless of which root was injected all the sprouts received some radioactivity, though the nearest were significantly favoured. It appears that sectorization is only relative and that the sectors of the stump which are fed preferentially by different roots may overlap. On the other hand, no significant evidence of any favouring of the most vigorous sprouts was discovered. 


\section{Références bibliographiques}

Aymard M., Fredon J.J., 1986. Etude des relations entre une racine et les rejets de la souche chez Castanea sativa Mill. Ann. Sci. For., 43 (3), 351-363.

Bedeneau M., Pages L., 1984. Répartition de la sève brute entre les jeunes rejets de bouleau étudiée à l'aide d'un phytocide. Ann. Sci. For., 41 (2), 237-248.

BerTHIER B., 1984. Fonctionnement d'un écosystème forestier : croissance, biomasse et productivité du compartiment ligneux épigé des taillis de châtaigniers (Castanea sativa Mill.) du Sud-Est de la France. Thèse de $3^{\mathrm{e}}$ cycle, Grenoble, $62 \mathrm{p}$.

Bовау V., 1985. Application d'un nouvelle méthode de mesure de la transpiration à un jeune taillis de châtaigniers Castanea sativa Mill. Mémoire de D.E.A., Université de Paris-Sud, 31 p.

Brochet P., Gerbier N., 1972. Une méthode pratique de calcul de l'évapotranspiration potentielle. Ann. Agron., 23 (1), 31-49.

Destremeau D.X., Roderbourg J., 1968. Répartition de la sève entre les rejets. Ann. Rech. For. Maroc, tome II, rapport 1968-1969, 237-242.

Granier A., 1985. Une nouvelle méthode pour la mesure du flux de sève brute dans le tronc des arbres. Ann. Sci. For., 42 (2), 193-200.

Ibrahim M., Rapp M., Berger A., Lossaint P., 1980. Evaluation de la transpiration d'un peuplement de Pinus pinea $\mathrm{L}$. en condition naturelle. Acta oecologica, Oecol. Plant., 1 (15), $\mathrm{n}^{\circ} 4,395-407$.

JoRdan C.F., Kune J.R., 1977. Transpiration of trees in a tropical rainforest. J. appl. Ecol., 14, 853-860.

Kline J.R., Martin J.R., Jordan C.F., Koranda J.J., 1970. Measurement of transpiration in tropical trees with tritiated water. Ecology, 51 (6), 273-284.

Kline J.R., Reed K.L., Waring R.H., Stewart M.L., 1976. Field measurement of transpiration in Douglas-fir. J. appl. Ecol., 13, 273-283.

Luvall J.C., Murphy C.E., 1982. Evaluation of the tritiated water method for measurement of transpiration in young Pinus taeda L. Forest Sci., 28 (1), 5-16.

Owston P.W., Smith J.L., Halverson H.G., 1970. Development of some radioisotope procedures for measuring water movement in trees. Isotopes and Radiation Technology, 7 (4), 396-401.

Owston P.W., Smith J.L., Halverson H.G., 1972. Seasonal water movement in tree stems. Forest science, 18, 266-272.

Riedacker A., 1973. Les taillis d'Eucalyptus au Maroc. Ann. Rech. For., Maroc, 13, 157-352.

Sokal. R.R., RohlF F.J., 1981. Biometry, Freeman and Co, San Francisco, 429-432. 